\title{
BMJ Open Effectiveness of a nurse educational oral feeding programme on feeding outcomes in neonates: protocol for an interrupted time series design
}

\author{
Sandrine Touzet, ${ }^{1,2}$ Anne Beissel, ${ }^{3}$ Angélique Denis, ${ }^{1}$ Fabienne Pillet, ${ }^{3}$ \\ Hélène Gauthier-Moulinier, ${ }^{3}$ Sophie Hommey, ${ }^{1}$ Olivier Claris, ${ }^{3,4}$ \\ The TOP Study Group
}

\begin{abstract}
To cite: Touzet S, Beissel A, Denis $A$, et al. Effectiveness of a nurse educational oral feeding programme on feeding outcomes in neonates: protocol for an interrupted time series design. BMJ Open 2016;6: e010699. doi:10.1136/ bmjopen-2015-010699

- Prepublication history for this paper is available online. To view these files please visit the journal online (http://dx.doi.org/10.1136/ bmjopen-2015-010699).
\end{abstract}

Received 10 December 2015 Revised 16 February 2016 Accepted 21 March 2016

\section{CrossMark}

${ }^{1}$ Hospices Civils de Lyon, Pôle Information Médicale Évaluation Recherche, Lyon F-69003, France

${ }^{2}$ Universite de Lyon, EA Laboratoire Health Services and Performance Research (HESPER) 7425, Lyon F-69008, France

${ }^{3}$ Hospices Civils de Lyon, Hopital Femme Mère Enfant, Department of Neonatal Intensive Care Unit, Bron F-69500, France

${ }^{4}$ Universite de Lyon, EA Santé Individu Société (SIS) 4128, Lyon F-69002, France

Correspondence to

Sandrine Touzet;

sandrine.touzet@chu-lyon.fr

\section{ABSTRACT}

Introduction: Oral feeding is a complex physiological process. Several scales have been developed to assess the ability of the neonate to begin suck feedings and assist caregivers in determining feeding advancement. However, feeding premature neonates remains an ongoing challenge and depends above all on caregivers' feeding expertise. We will evaluate the effect of a nurse training programme on the achievement of full oral feeding with premature neonates.

Methods and analysis: The study design will be an interrupted time series design with 3 phases: (1) A 6month baseline period; (2) a 22-month intervention period and (3) a 6-month postintervention period. The intervention will consist of an educational programme, for nurses and assistant nurses, on feeding patterns in neonates. The training modules will be composed of a 2-day conference, 2 interactive multidisciplinary workshops, and routine practice nurse coaching. A total of 120 nurses and 12 assistant nurses, who work at the neonatal unit during the study period, will participate in the study. All premature neonates of $<34$ weeks postmenstrual age (PMA) will be included. The primary outcome will be the age of tube withdrawal PMA and chronological age are taken into account. The secondary outcomes will be the transition time, length of hospital stay, competent suckle feeding without cardiorespiratory compromise, rate of neonates presenting with feeding issues or feeding rejection signs, and current neonatal pathologies or deaths during hospital stay. A segmented regression analysis will be performed to assess the impact of the programme.

Ethics and dissemination: Approval for the study was obtained from the Hospital Ethics Committee, and the Institutional Review Board, as well as the French Data Protection Agency. The findings from the study will be disseminated through peer-reviewed journals, national and international conference presentations and public events.

Trial registration number: NCT02404272 (https:// clinicaltrials.gov).

\section{Strengths and limitations of this study}

- This educational nurse programme is designed to include maximum participation of interested caregivers in neonatal feeding patterns.

- A coordinated multidisciplinary diagnostic and management approach will be implemented through the creation of an individualised feeding protocol combined with feeding specialists coaching.

- Moreover, we will designate 'local opinion leaders', otherwise experts in our study.

- The study aims to show that interactive workshops can result in considerable changes in professional practice.

- However, methods to implement continuous medical education for paramedical professions still need to be developed.

\section{INTRODUCTION}

\section{Background and rationale}

Poor oral-motor developments in neonates are common ${ }^{1}$ and the prevalence of feeding dysfunctions in former premature infants is twice that of full-term born infants. ${ }^{2}$ Oral feeding is a complex physiological process that depends primarily on the coordination of sucking, swallowing and respiration. ${ }^{3}$ Full term born children demonstrate such skills at birth. However, premature infants may experience delayed transition to independent oral feeding, which exposes them to the possibility of long-term tube feeding. ${ }^{4}$ There are several implications to delayed transition, such as delayed oral feeding autonomy, intrusive medical oral sphere care and impaired eating disorders, potentially resulting in delayed hospital discharge and increased financial burdens. ${ }^{56}$

Different interventions have shown their efficacy in accelerating transition from tube 
to full oral feeding, which can also reduce the duration of hospital stay. Non-nutritive sucking through the utilisation of a pacifier accelerates transition to full oral feeding and allows earlier tube withdrawal, while decreasing hospitalisation time. ${ }^{7}$ Early introduction to suck feedings also accelerates transition from tube to oral feeding and allows for earlier tube removal. ${ }^{8} 9$ Prefeeding oral and perioral stimulations (cheeks, lips, jaw, gums and tongue) result in earlier achievement of full oral feeding without noticeable impact on length of stay. ${ }^{10}{ }^{11}$ Finally, oral-motor interventions (assisted movement to activate muscle contraction, and movement against resistance to build strength) prior to the introduction of oral feedings are also found to reduce the transition to full oral feeding autonomy. ${ }^{12}$

Yet, as Kish ${ }^{13}$ shows, oral feeding readiness is affected by adequate suck-swallow-breathe coordination and depends on gestational age, behavioural state organisation and physiological stability, as well as being influenced by caregivers and the environment. Several scales have been developed to assess the ability of neonates to start suck feedings and assist caregivers in determining feeding advancement. ${ }^{4}{ }^{13-16}$ However, in spite of existing guidelines, feeding premature neonates remains an ongoing challenge and relies on caregivers' feeding expertise, often requiring multidisciplinary guidance. ${ }^{17}$ Negative oral stimulation as well as overzealous feeding practices may induce oral-motor disorganisation, potentially causing defensive feeding behaviour, and hinder the differentiation of feeding skills. $^{18} 19$

We hypothesise that an oral feeding training programme dedicated to the caregivers of a neonatal unit could (1) help the caregivers to reflect on their current feeding practices and enhance their diagnostic and management approach of infants presenting with feeding difficulties, (2) reduce the length of hospital stay for neonates and (3) reduce the rate of neonates presenting with oral-motor dysfunction or defensive feeding behaviour.

\section{Aims of the study}

The purpose of this study is to evaluate the impact of an educational oral feeding nurse training programme on the achievement of full oral feeding and transition time (initiation to full oral feeding) among all admitted neonates of $<34$ weeks postmenstrual age (PMA).

Caregivers' theoretical knowledge and practical feeding skills will be evaluated, as well as their satisfaction level with the educational oral feeding programme.

\section{METHODS/DESIGN}

\section{Study design}

The study design will be an interrupted time series design with three phases: (1) a 6-month baseline period; (2) a 22-month intervention period and (3) a 6-month postintervention period.

\section{Setting and participants}

The setting of this study is a 44-bed, level IIIC, Neonatal Intensive Care Unit (NICU) ${ }^{20}$ of the Femme Mere Enfant Hospital, Bron, Lyon, France.

All nurses and assistant nurses (a total of 120 nurses and 12 assistant nurses) of the neonatal unit will participate in the study. All premature neonates of $<34$ weeks PMA, admitted to the unit during the study period, will be included. PMA will be used as the gestational age and postnatal age in weeks. Neonates who will have surgery with endotracheal intubation will not be included. Neonates will not be included if one of the parents refuse his/her participation in the study.

\section{Group at baseline period}

The baseline group will comprised premature infants who were hospitalised prior to the initiation of the educational nurses programme, at which time non-nutritive sucking is applied intermittently. Oral stimulations and oral-motor rehabilitation is performed during the baseline period by physiotherapists and speech therapists as it is usually done on infants presenting with feeding issues. Oral feeding is initiated between 32 and 34 weeks of PMA. No feeding protocol will be in place at the time, and parents participate in the feedings when present.

\section{Intervention}

The intervention will consist of an educational programme, for nurses and assistant nurses, on feeding patterns in neonates. Training modules will be composed of a 2-day conference, two interactive multidisciplinary workshops and routine practice nurse coaching (table 1).

The content of the trainings will be developed by a group of experts and adapted to nurse competency levels. A protocol leaflet will be produced for the personnel. The expert group will comprised two nurses specialised in developmental care, a speech therapist, a physiotherapist, a psychologist and a neonatologist. All the experts work in the neonatal unit. Six training modules will be scheduled over a 22-month period to include the group of 120 nurses and 12 nurse assistants from the unit.

\section{Training modules}

Each training module will last 4 months and will consist of a 2-day conference, held during the first month, followed by two multidisciplinary workshops. Routine practice nurse coaching will take place throughout this study period.

1. The conference will provide theoretical knowledge about feeding patterns in neonates. Anatomy, physiology and development of feeding milestones will be analysed. Different interventions, shown to accelerate the transition from tube to full oral feeding, will be presented. The impact of both environment and caregiver behaviour on feeding success will be evaluated. Feeding difficulty symptoms, patient 
Table 1 Educational training programme

\begin{tabular}{|c|c|c|c|c|}
\hline $\begin{array}{l}\text { Multidisciplinary } \\
\text { educational } \\
\text { programme }\end{array}$ & Theoretical conference & $\begin{array}{l}\text { Interactive } \\
\text { workshop } 1\end{array}$ & $\begin{array}{l}\text { Interactive } \\
\text { workshop } 2\end{array}$ & Routine practice nurse coaching \\
\hline Content & $\begin{array}{l}\text { Theoretical knowledge } \\
\text { about feeding pattern } \\
\text { Introduction of the oral } \\
\text { feeding protocol }\end{array}$ & Video recordings & Video recordings & $\begin{array}{l}\text { Routine feeding practice } \\
\text { Oral feeding protocol } \\
\text { Detection of infants presenting with } \\
\text { feeding issues }\end{array}$ \\
\hline Speech therapist & Instructor & Instructor & Instructor & Instructor \\
\hline Physiotherapist & Instructor & Instructor & Instructor & Instructor \\
\hline Paediatrician & Instructor & Instructor & & \\
\hline Psychologist & Instructor & & Instructor & Instructor \\
\hline Length & 2 days & $2 \mathrm{~h}$ & $2 \mathrm{~h}$ & During the entire study period \\
\hline Time frame & 4 months & & & \\
\hline
\end{tabular}

management and feeding outcomes will also be reviewed, and feedback from the different experts regarding feeding patterns will be presented at the conference, at which time the standardised individualised feeding protocol will be introduced.

2. The multidisciplinary interactive workshops will consist of video presentations of neonatal bottle or breast feedings performed in the neonatal unit. Video presentations will demonstrate oral and perioral stimulation, non-nutritive sucking, oral-motor re-education and feedings. These workshops will focus on nursing neonate feeding practices in a multidisciplinary setting. Difficulties encountered during feedings, such as overzealous as well as passive nurse feeding behaviours, will be discussed, and optimal position and posture during feeding sessions will be evaluated. The standardised, individualised feeding protocol and its application to caregivers will be considered. Two members of the expert group will be present and lead workshop discussions.

3. The routine practice nurse coaching will be performed by a speech therapist and physiotherapist present at the neonatal unit. They will support nurses and assistant nurses in their routine feeding practice and application of feeding protocols. They will also assist caregivers with early detection of infants presenting with feeding issues, and help them apply the oral-motor rehabilitation protocol when necessary.

\section{The standardised individualised feeding protocol}

The standardised feeding protocol will be applied to neonates who present with physiologically stable conditions. The nurse will evaluate the infant's medical condition prior to the feeding and determine if the intervention will be possible. Infants with unstable cardiorespiratory function will not get the intervention. Unstable cardiorespiratory function will be defined as: more than one apnoea and/or bradycardia per hour (breathing pauses that last for $>20 \mathrm{~s}$ or for $>10 \mathrm{~s}$ if associated with bradycardia $<80 / \mathrm{min}$ or oxygen desaturation), high oxygen use or tachypnoea (respiratory rate
$>60 / \mathrm{min})$, uncontrolled infection or haemodynamic support. Neonates' sleeping periods will be respected. If the neonate presents with signs of feeding rejection or symptoms of feeding difficulty, the intervention will be stopped and resumed at a later time. Rejection signs or feeding difficulties will be defined as no demonstration of hunger cues, bottle refusal, coughing, choking or vomiting during feedings, hypoxia or unstable heart rate during feeding sessions; feeding intake exceeding $30 \mathrm{~min}$, excitement, discomfort or arching of the back during feedings, and gagging or falling asleep during feedings. With infants of at least 32 weeks PMA, parents will be able to participate in non-nutritive sucking as well as the feedings, with coaching by the bedside nurse.

Emphasis will be placed on optimal position and posture during feeding sessions and minimisation of negative environmental stimulations, such as stress, lighting and noise. The bedside nurse will keep a feeding record on oral feeding advancement (timing of feeding, amount of feeding, feeding difficulties, feeding progress). Each participating nurse and assistant nurse will have direct access to our protocol leaflet. Our educational nurse programme includes a combination of strategies shown to be effective in accelerating transition from tube to full oral feeding: non-nutritive sucking, oral and perioral stimulation, early sucking introduction for all neonates in the unit, as well as oral-motor interventions for infants presenting with feeding difficulties (figures 1 and 2).

\section{Implementation strategies}

Implementation support strategies will be introduced to facilitate the intervention. These methods consist of 'expert' feeding rounds that will take place on a regular basis of one round per day for the physiotherapist and one round per week for the speech therapist and the paediatrician. These expert feeding rounds will have multiple aims: (1) reinforce the key messages of the standardised feeding protocol, (2) monitor nurses' and assistant nurses' compliance with the feeding protocol (3) identify the neonates for whom the feeding protocol 


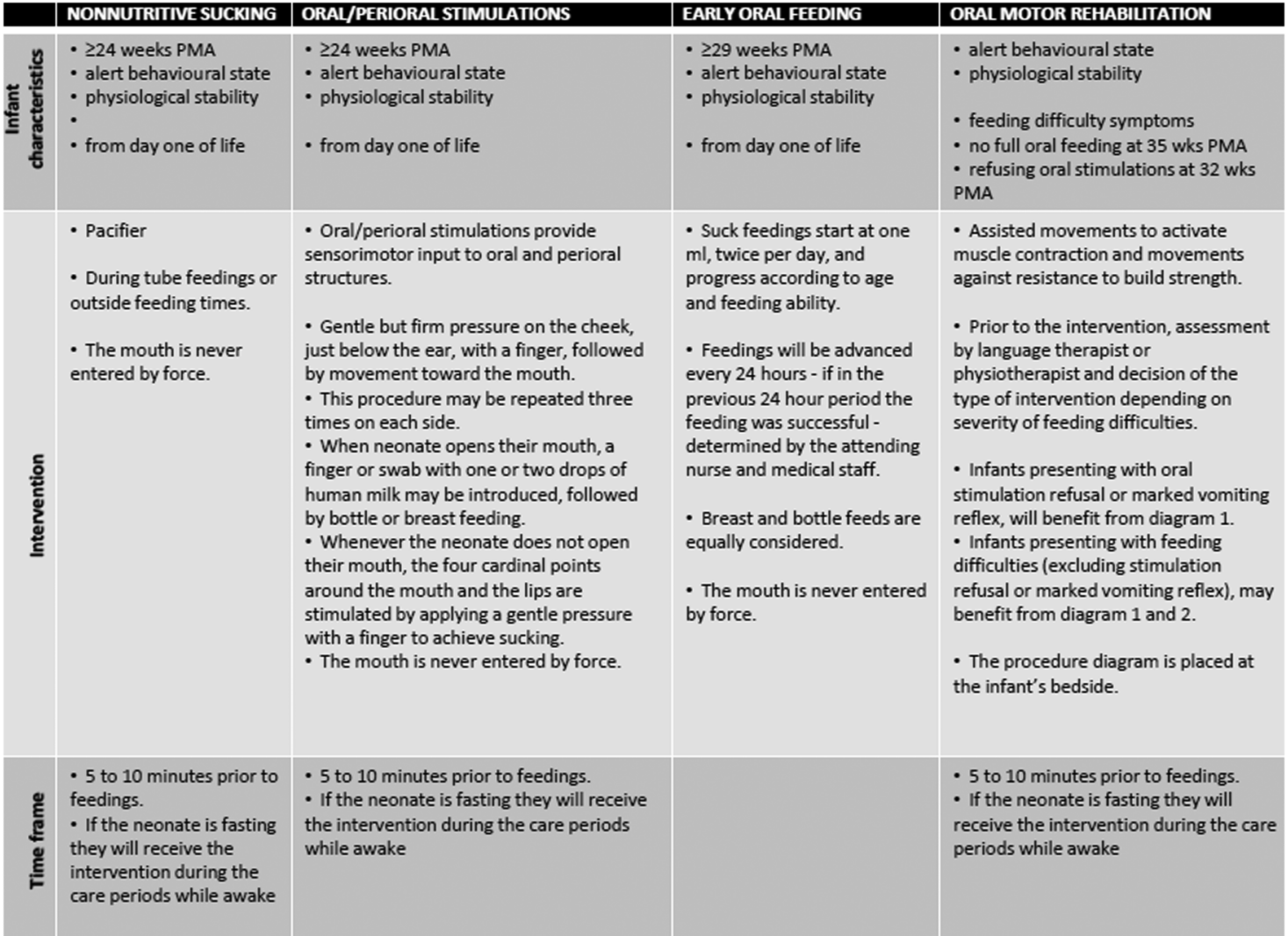

Figure 1 The standardised individualised feeding protocol. This figure describes the oral and perioral stimulation gestures. PMA, postmenstrual age.

is not applied correctly and (4) engage provider-focused feeding discussions.

\section{Outcomes measures}

The primary outcome to be measured will be the age of tube withdrawal. PMA will be described in weeks. Chronological age (CA) will be described in days, weeks or months. ${ }^{21}$ Data collection for the neonates will be obtained through their medical files: PMA will be determined from obstetrical records.

The secondary outcomes are described below:

- Transition time, which will be defined as the time frame between the start of oral feeding, and full oral feeding autonomy (date of tube removal). PMA and CA will be considered.

- Length of hospital stay.

- Competent suckle feeding without cardiorespiratory compromise (PMA and CA will be considered). Cardiorespiratory compromise during suckle feeding will be defined by apnoea and/or bradycardia (breathing pauses that last for $>20 \mathrm{~s}$ or for $>10 \mathrm{~s}$ if associated with bradycardia $<80 \mathrm{bpm}$ for $10 \mathrm{~s}$ or oxygen desaturation) occurring up to $30 \mathrm{~min}$ after feedings, and at least at two meals per day after tube removal.

- Current neonatal pathologies during hospital stay: bronchopulmonary dysplasia, necrotising enterocolitis, periventricular leucomalacia, intraventricular haemorrhage, hypoxic ischaemic encephalopathy, retinopathia of the neonate, culture proven bloodstream infections.

- Deaths during hospital stay.

\section{Process measures}

- Nurses' involvement in the training modules will be evaluated through their presence at the training sessions.

- Caregivers' knowledge acquisition and satisfaction scores will be assessed through a questionnaire following each training module.

- Time dedicated to routine practice nurse coaching (also called feeding rounds) in their routine feeding practice and application of feeding protocols will be measured.

- Nurses' protocol compliance: to assess nurses' protocol compliance, a questionnaire will be developed by health providers working at the neonatal unit, and will be revised by questionnaire design experts. The instruments' question and response categories will be phrased in simple terms. They will be tested for comprehensibility by health providers from the neonatal unit who are not participating in the study protocol.

\section{Balancing measures}

Feeding difficulties or feeding rejection signs, defined in our study as no demonstration of hunger cues, bottle 
Figure 2 Oral-motor

rehabilitation. Infants presenting

with an oral stimulation refusal or

marked vomiting reflex will benefit

from the oral-motor rehabilitation

protocol described in diagram

1. Infants presenting with feeding

difficulties (excluding the

stimulation refusal or marked

vomiting reflex) will benefit from

the oral-motor rehabilitation

protocol described in diagrams 1

and 2.
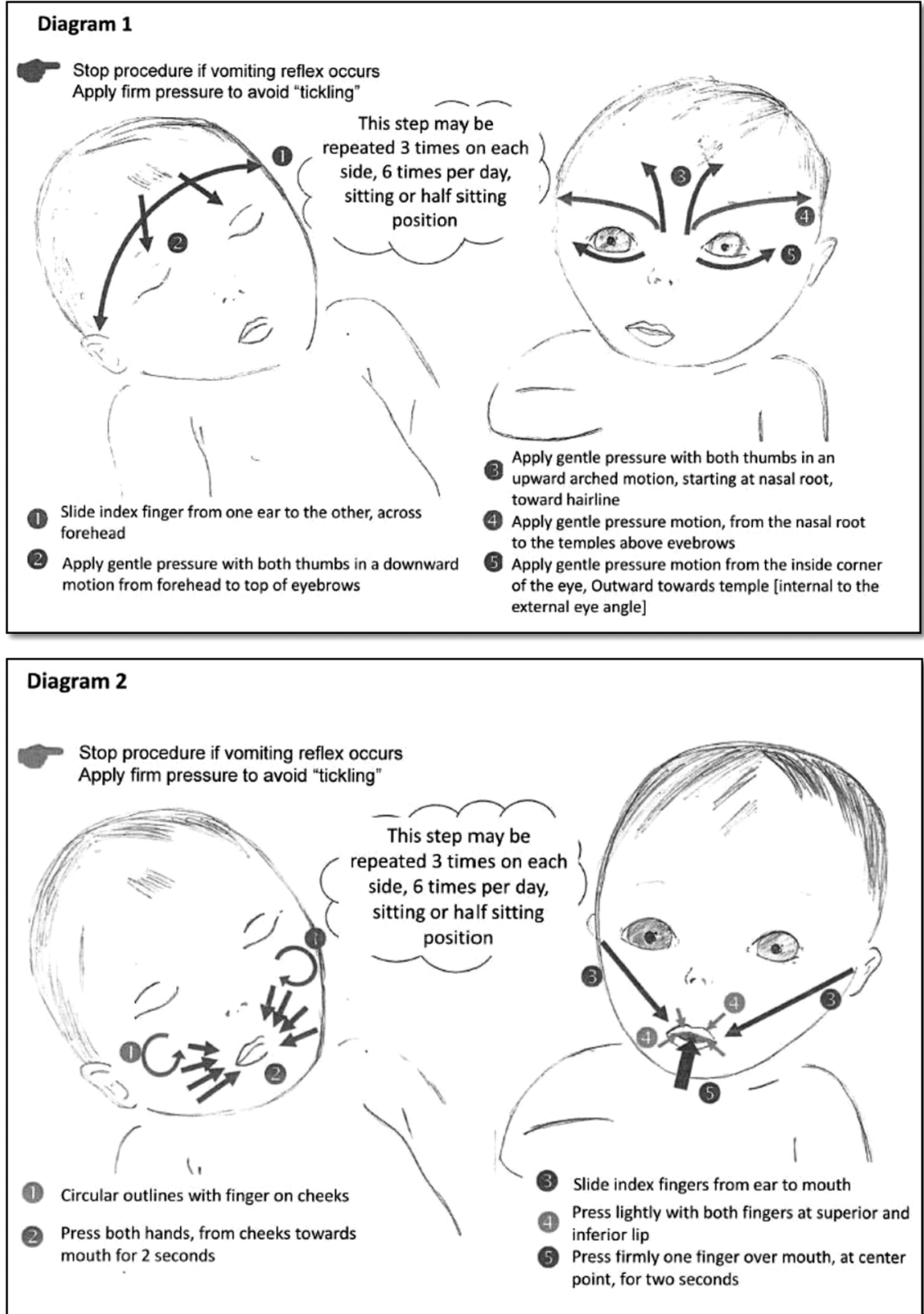

refusal, coughing, choking or vomiting during feedings, hypoxia or unstable heart rate during feeding sessions, feeding intake exceeding $30 \mathrm{~min}$, excitement, discomfort or arching of the back during feedings, and gagging or falling asleep during feedings.

\section{Other changes occurring in the NICU during the study period}

All events, such as leadership changes, and protocol modifications that occur during the study period and may interact with the intervention or the study results will be recorded in a logbook.

\section{Blinding}

Owing to the nature of the intervention, healthcare providers, parents and researchers will not be blinded to the intervention phase.

\section{Study sample size}

According to a pilot study, the mean age of tube withdrawal is $35.7 \pm 2$ weeks PMA (data unpublished). We aim to detect a decrease of 5 days in the mean age of tube withdrawal. A sample size of 130 neonates in the baseline period and the postintervention period will be needed to detect this difference with a power of $80 \%$ and $5 \%$ risk of type I error.

The anticipated difference in outcome measure is based on the expected effectiveness of our educational programme and data from previous studies. ${ }^{9} 1122$

On the basis of the census data, it is anticipated that we will be able to recruit 20-25 new patients from 24 to 34 weeks PMA into the proposed study each month, which means that 138 premature infants may be included during the 6-month pre-educational programme period; 506 during the 22-month training 
period, and 138 during the 6-month posteducational programme. A total of 680 premature infants may be included during the 34-month study period.

In interrupted time series studies, sample size calculations are related to the estimation of the number of observations or time points at which data will be collected. A sufficient number of time points before and after the intervention is needed to conduct a segmented regression analysis: a study of 34 months meets these conditions. ${ }^{23}$

\section{Statistical considerations}

Data analysis will be performed by the data management and analysis centre using SAS/STAT software.

Statistical analysis will include the following steps:

- A descriptive and bivariate comparative study of the characteristics of the newborns, between the three periods of study (gestational age at birth; PMA, CA when neonates present with feeding difficulties; age (PMA, CA) at the initiation of bottle/breast feedings; age (PMA, CA) at full oral feeding autonomy; bloodstream infections; mortality; length of stay).

- A bivariate comparative study of the primary and the secondary outcomes between the baseline period and the postintervention period.

- A graphical analysis of the average age (PMA; CA) of tube withdrawal, by month, according to the method of statistical process control. ${ }^{24}$

- A multivariate analysis by the method of segmented regression analysis to study the impact of training on the average age (PMA, CA) of tube removal by month. ${ }^{23}$ The statistics of Durbin-Watson will be calculated to test the hypothesis of non-independence between the observations. Where appropriate, the segmented regression model will be adjusted to the autocorrelation parameters. An adjustment may be made on other aggregate covariates such as the number of professionals trained in oral and perioral stimulation gestures by month.

The secondary end points will be analysed with the same statistical method.

A descriptive analysis of the average scores of professional self-assessment of learning following training, and a descriptive analysis of the average level of satisfaction with the training module.

\section{Dissemination}

Healthcare staff consent was obtained prior to the study. As stipulated by French law, given the study design (lack of randomisation) and type of intervention (educational programme on recognised clinical practices), an instruction sheet will be given to the parents who will be permitted to refuse the inclusion of their infant in the study.

The results of our study will be disseminated on the Neonatology' unit's website. Additional dissemination will occur through presentations at conferences, such as neonatology conferences, regionally and nationally, and through articles published in peer-reviewed journals.
Presentations at International conferences as 'Hot Topics in Neonatology 2016' and national conferences as 'Journées Françaises de Néonatologie (JFRN)' will take place from December 2016. Study results will be submitted from December 2016 in peer-reviewed journals.

\section{DISCUSSION}

\section{Discussion of the intervention}

In 1998, the American Academy of Pediatrics has established guidelines on discharge of high-risk neonates, including preterms. One of the three competencies recognised as essential before hospital leave is adequate oral feeding to support appropriate growth. However, no guidelines exist on clinical pathways for acquiring these feeding competencies. ${ }^{25}$

A literature review demonstrates significant research on feeding development interventions, assessment of feeding readiness and feeding advancement algorithms. Different strategies have been shown to achieve earlier tube weaning: non-nutritive sucking, ${ }^{7}$ oral and perioral stimulation, ${ }^{10}$ 11 oral-motor intervention ${ }^{12}$ and early introduction of suck feeding. ${ }^{8}{ }^{9}$ These methods are effective, either individually or in combination, to accelerate transition to independent oral feeding. ${ }^{10} 2226$ Our educational nurse programme includes a combination of these strategies: non-nutritive sucking, oral and perioral stimulation, early sucking introduction for all neonates in the unit, as well as oral-motor interventions for infants presenting with feeding difficulties.

Oral Feeding readiness assessment scales have been established to evaluate neonates' ability to start suck feedings as well as detect infants presenting with suck difficulties. ${ }^{4} 15 \quad 27$ Researchers have also shown that protocol-driven clinical pathways permit earlier oral feeding autonomy. ${ }^{9} 28$

However, these programmes are more effective when delivered by knowledgeable and skilled nurses, with the assistance of experienced feeding specialists. ${ }^{19} 29 \quad 30$ Similarly, infants presenting with feeding difficulties should be supported by individualised protocols. ${ }^{30}$ Parent involvement in feedings is also important in maternal infant attachment and parent well-being. ${ }^{18} 1931$ However, if not well controlled, this aspect could raise the anxiety level of already fragile parents. ${ }^{32}$

This educational nurse programme contains essential elements to be effective. It will be coordinated by an expert group and designed to include maximum participation of interested caregivers in neonatal feeding patterns (nurses, assistant nurses, physiotherapists, language speech therapist, psychologist, neonatologists). The individualised feeding protocol will be adapted to daily practice in a multidisciplinary setting, utilising video recordings of neonatal feeding sessions. The protocol will be implemented in daily practice with coaching from physiotherapists and a speech language therapist. Parents can participate in non-nutritive sucking, and 
feedings, with infants of at least 32 weeks PMA, with coaching from the bedside nurse.

Studies show that effective nurse training benefits from: (1) involvement of experts, considered by teams as 'local opinion leaders', ${ }^{33}$ (2) interactive continuing medical education through real case studies, where better learning outcomes have been demonstrated compared with more traditional teaching, ${ }^{34} 35$ and (3) coordinated multidisciplinary diagnostic and management approach.

The study aims to show that interactive workshops can result in considerable changes in professional practice. Moreover, we will designate 'local opinion leaders', otherwise experts in our study. A coordinated multidisciplinary diagnostic and management approach will be implemented through the creation of an individualised feeding protocol combined with feeding specialists coaching. However, methods to implement continuous medical education for paramedical professions still need to be developed. ${ }^{36}$

\section{Discussion of study design}

The interrupted time series design includes a data sequence before and after the implementation of the intervention. ${ }^{37}{ }^{38} \mathrm{An}$ interrupted time series design is an effective quasiexperimental approach for evaluating effects of interventions that are introduced at a specific point in time when a randomised controlled trial is not feasible. ${ }^{39} 40$ The analysis of a time series allows us to assess the initial value of the criteria of judgement (intercept), the underlying trend (slope) and the sustainability of the impact of the intervention. ${ }^{41}$ This design is effective if a fairly large number of measurements for the outcome of interest are available and a segmented regression can be used to analyse the data. ${ }^{42}$ The Effective Practice and Organisation of Care (EPOC) group recommends a minimum of three points before and six points after surgery. The intervention should be in place at one time or a specified period, and the measures of criteria must be objective. ${ }^{43}$

Interrupted time series analysis depends on the availability of accurate and quality data. ${ }^{44}$ In our study, data will be routinely collected in the computerised clinical records of newborns, ensuring successful execution of the study, and the collection of a sufficient number of measurements.

To utilise the strength of this design, a modification to standard regression analysis, such as segmented regression, is required. Segmented regression analysis is a powerful statistical method for evaluating intervention effects in interrupted time series studies. ${ }^{40} 45$ Segmented regression can identify sudden change due to an intervention, as well as more gradual changes over time. ${ }^{42}$

We selected this study design due to its methodological quality and feasibility. One advantage is that it will permit us to separate results of individual training programmes and progression of underlying practices. ${ }^{46}$ It will allow us to consider the secular trend (change in epoch). Indeed, we will analyse neonates' data during a 6-month period preceding the implementation phase. This baseline period will give us a temporal control that will help us to determine whether the particular intervention has a significantly greater effect than the underlying secular trend. Moreover, this type of study allows us to measure the learning progression of neonatal feeding patterns by caregivers. Effectively, we will establish a learning curve on feeding techniques and protocol compliance. However, the biggest threat to time series analysis is events occurring at the same time as the intervention and producing similar effects (ie, history) ${ }^{47}$ One of the more notable strengths of interrupted time series studies is the intuitive graphical presentation of results. The first step when analysing data over time is the visual inspection of the series data. ${ }^{48}$ This graphical representation is a useful tool for caregivers to visualise the impact of the training programme.

Acknowledgements The authors are grateful to Mrs Anne-Sophie Magrou and Caroline Keita for their contribution in describing the standardised feeding protocol and reviewing the manuscript. They also thank Mrs Catarina de Carvalho for her help with the English.

Collaborators The TOP study group: Anne Beissel, Celine Bellet, Olivier Claris, Isabelle Conti, Angélique Denis, Fabienne Deruy, Fabienne Doiret, Laetitia Fernandez, Hélène Gauthier Moulinier, Anne-Sophie Goyet, Sophie Hommey, Caroline Keita, Sophie Laborie, Marie-Annick Le Pogam, Cateina Maggi-Perpoint, Anne-Sophie Magrou, Pauline Occelli, Fabienne Pillet, Franck Plaisant, Helene Tarifa-Buisson, Sandrine Touzet.

Contributors All the authors have made substantial contributions to the development of this study. FP conceived this study with $A B$ and designed it with $S T, A D, H G M, S H$ and $O C$. AD and ST contributed to the statistical plan and are responsible for statistical analysis. $A D$ and $S H$ are responsible for the data management. ST, $A B, F P$ and $A D$ have contributed to the development of this protocol. ST wrote the first draft of the manuscript. All the authors read and approved the final manuscript.

Funding This study is supported by a grant from the Programme Hospitalier de Recherche Infirmière et Paramédicale (PHRIP) 2012 of the French Ministry of Health (Ministère chargé de la Santé, Direction de l'Hospitalisation et de l'Organisation des Soins).

Disclaimer The funder has no role in the study design, data collection, data analysis, decision to publish or writing of the manuscript.

Competing interests None declared.

Patient consent Obtained.

Ethics approval Approval for the study was obtained from the Hospital Ethics Committee, the Sud Est IV Institutional Review Board, as well as the French Data Protection Agency.

Provenance and peer review Not commissioned; externally peer reviewed.

Data sharing statement All data from the study will be available for all TOP study investigators. All data will be published.

Open Access This is an Open Access article distributed in accordance with the Creative Commons Attribution Non Commercial (CC BY-NC 4.0) license, which permits others to distribute, remix, adapt, build upon this work noncommercially, and license their derivative works on different terms, provided the original work is properly cited and the use is non-commercial. See: http:// creativecommons.org/licenses/by-nc/4.0/

\section{REFERENCES}

1. Jadcherla SR, Wang M, Vijayapal AS, et al. Impact of prematurity and co-morbidities on feeding milestones in neonates: a retrospective study. J Perinatol 2010;30:201-8. 
2. Mercado-Deane MG, Burton EM, Harlow SA, et al. Swallowing dysfunction in infants less than 1 year of age. Pediatr Radiol 2001;31:423-8.

3. Lau C, Smith EO, Schanler RJ. Coordination of suck-swallow and swallow respiration in preterm infants. Acta Paediatr 2003;92:721-7.

4. Thoyre SM, Shaker CS, Pridham KF. The early feeding skills assessment for preterm infants. Neonatal Netw 2005;24:7-16.

5. Schmitt SK, Sneed L, Phibbs CS. Costs of newborn care in California: a population-based study. Pediatrics 2006;117:154-60.

6. Rommel N, De Meyer A, Feenstra L, et al. The complexity of feeding problems in 700 infants and young children presenting to a tertiary care institution. J Pediatr Gastroenterol Nutr 2003;37:75-84.

7. Pinelli J, Symington A. Non-nutritive sucking for promoting physiologic stability and nutrition in preterm infants. Cochrane Database Syst Rev 2005;(4):CD001071.

8. Pridham KF, Sondel S, Chang A, et al. Nipple feeding for preterm infants with bronchopulmonary dysplasia. J Obstet Gynecol Neonatal Nurs 1993;22:147-55.

9. Simpson C, Schanler RJ, Lau C. Early introduction of oral feeding in preterm infants. Pediatrics 2002;110:517-22.

10. Arvedson J, Clark H, Lazarus $\mathrm{C}$, et al. Evidence-based systematic review: effects of oral motor interventions on feeding and swallowing in preterm infants. Am J Speech Lang Pathol 2010;19:321-40.

11. Fucile S, Gisel E, Lau C. Oral stimulation accelerates the transition from tube to oral feeding in preterm infants. J Pediatr 2002;141:230-6.

12. Lessen BS. Effect of the premature infant oral motor intervention on feeding progression and length of stay in preterm infants. Adv Neonatal Care 2011;11:129-39.

13. Kish MZ. Oral feeding readiness in preterm infants: a concept analysis. Adv Neonatal Care 2013;13:230-7.

14. Bingham PM, Ashikaga T, Abbasi S. Relationship of neonatal oral motor assessment scale to feeding performance of premature infants. J Neonatal Nurs 2012;18:30-6.

15. Fujinaga $\mathrm{Cl}$, de Moraes SA, Zamberlan-Amorim NE, et al. Clinical validation of the Preterm Oral Feeding Readiness Assessment Scale. Rev Lat Am Enfermagem 2013;21 Spec No:140-5.

16. Crowe $L$, Chang $A$, Wallace $K$. Instruments for assessing readiness to commence suck feeds in preterm infants: effects on time to establish full oral feeding and duration of hospitalisation. Cochrane Database Syst Rev 2012;4:CD005586.

17. Hawdon JM, Beauregard N, Slattery J, et al. Identification of neonates at risk of developing feeding problems in infancy. Dev Med Child Neurol 2000;42:235-9.

18. Als $\mathrm{H}$, Lawhon $\mathrm{G}$, Brown $\mathrm{E}$, et al. Individualized behavioral and environmental care for the very low birth weight preterm infant at high risk for bronchopulmonary dysplasia: neonatal intensive care unit and developmental outcome. Pediatrics 1986;78:1123-32.

19. Ross ES, Philbin MK. Supporting oral feeding in fragile infants: an evidence-based method for quality bottle-feedings of preterm, ill, and fragile infants. J Perinat Neonatal Nurs 2011;25:349-57.

20. Stark AR, American Academy of Pediatrics Committee on Fetus and Newborn. Levels of neonatal care. Pediatrics 2004;114:1341-7.

21. Engle WA, American Academy of Pediatrics Committee on Fetus and Newborn. Age terminology during the perinatal period. Pediatrics 2004:114:1362-4.

22. Rocha AD, Moreira MEL, Pimenta HP, et al. A randomized study of the efficacy of sensory-motor-oral stimulation and non-nutritive sucking in very low birthweight infant. Early Hum Dev 2007;83:385-8.

23. Wagner AK, Soumerai SB, Zhang F, et al. Segmented regression analysis of interrupted time series studies in medication use research. Clin Pharm Ther 2002;27:299-309.

24. French Health Authority. Statistical process control. 2004:92. http:// www.has-sante.fr/portail/upload/docs/application/pdf/2009-10/ maitrise_statistique_des_processus_en_sante_guide_2005.pdf

25. Gennattasio A, Perri EA, Baranek D, et al. Oral feeding readiness assessment in premature infants. MCN Am J Matern Child Nurs 2015;40:96-104; E9-10

26. Pimenta HP, Moreira ME, Rocha AD, et al. Effects of non-nutritive sucking and oral stimulation on breastfeeding rates for preterm, low birth weight infants: a randomized clinical trial. J Pediatr (Rio J) 2008;84:423-7.
27. Palmer MM, Crawley K, Blanco IA. Neonatal Oral-Motor Assessment scale: a reliability study. J Perinatol 1993;13:28-35.

28. Kirk AT, Alder SC, King JD. Cue-based oral feeding clinical pathway results in earlier attainment of full oral feeding in premature infants. $J$ Perinatol 2007;27:572-8.

29. http://www.asha.org/policy/KS2004-00080/\#r4

30. Marcellus L, Harrison A, Mackinnon K. Quality improvement for neonatal nurses, part II: using a PDSA quality improvement cycle approach to implement an oral feeding progression guideline for premature infants. Neonatal Netw 2012;31:215-22.

31. Harding C. An evaluation of the benefits of non-nutritive sucking for premature infants as described in the literature. Arch Dis Child 2009;94:636-40.

32. Lee TY, Lee TT, Kuo SC. The experiences of mothers in breastfeeding their very low birth weight infants. J Adv Nurs 2009;65:2523-31.

33. Flodgren G, Parmelli E, Doumit G, et al. Local opinion leaders: effects on professional practice and health care outcomes. Cochrane Database Syst Rev 2011;(8):CD000125.

34. Davis D, O'Brien MAT, Freemantle N, et al. Impact of formal continuing medical education. Do conferences, workshops, rounds, and other traditional continuing education activities change physician behavior or health care outcomes? JAMA 1999;282:867-74.

35. Thomson O'Brien MA, Freemantle N, Oxman AD, et al. Continuing education meetings and workshops: effects on professional practice and health care outcomes. Cochrane Database Syst Rev 2001;(2): CD003030.

36. Gallagher L. Continuing education in nursing: a concept analysis. Nurse Educ Today 2007;27:466-73.

37. Cook TD, Campbell DT. Quasi-experiments: interrupted time-series designs in quasi-experimentation: design and analysis issues for field settings. Houghton Mifflin Company, 1979.

38. Ramsey CR, Matowe L, Grilli R, et al. Interrupted time series designs in health technology assessment: lessons from two systematic reviews of behaviour change strategies. Int J Technol Assess Health Care 2003;19:613-23.

39. Shadish WR, Cook TD, Campbell DT. Quasi-experiments: interrupted time-series designs. Experimental and Quasi-Experimental Designs for Generalised Causal Inference. Boston: Houghton Mifflin, 2002:171-206.

40. Taljaard M, McKenzie JE, Ramsay CR, et al. The use of segmented regression in analysing interrupted time series studies: an example in pre-hospital ambulance care. Implement Sci 2014;9:77.

41. Hanbury A, Farley K, Thompson C, et al. Immediate versus sustained effects: interrupted time series analysis of a tailored intervention. Implement Sci 2013;8:130.

42. Hopewell S, Ravaud P, Baron G, et al. Effect of editors' implementation of CONSORT guidelines on the reporting of abstracts in high impact medical journals: interrupted time series analysis. BMJ 2012;344:e4178.

43. Bero L, Grilli R, Grimshaw JM, et al. The Cochrane Effective Practice and Organisation of Care Group (EPOC) Module. Oxford: The Cochrane Library, 2002 Issue 1. Update Software.

44. Fretheim A, Soumerai SB, Zhang F, et al. Interrupted time-series analysis yielded an effect estimate concordant with the cluster-randomized controlled trial result. J Clin Epidemio 2013;66:883-7.

45. Lépée C, Klaber RE, Benn J, et al. The use of a consultant-led ward round checklist to improve paediatric prescribing: an interrupted time series study. Eur J Pediatr 2012;171:1239-45.

46. Bastuji-Garin S, Sbidian E, Gaudy-Marqueste C, et al, European Dermatology Network (EDEN). Impact of STROBE statement publication on quality of observational study reporting: interrupted time series versus before-after analysis. PLOS ONE 2013;8:e64733.

47. Bussières $A E$, Sales $A E$, Ramsay $T$, et al. Impact of imaging guidelines on X-ray use among American provider network chiropractors: interrupted time series analysis. Spine $J$ 2014;14:1501-9.

48. Colombet I, Sabatier B, Gillaizeau F, et al. Long-term effects of a multifaceted intervention to encourage the choice of the oral route for proton pump inhibitors: an interrupted time-series analysis. Qual Saf Health Care 2009;18:232-5. 